\title{
Accuracy in the Division of Acetaminophen Suppositories
}

\author{
Carlo Valerio Bellieni ${ }^{*}$, Elena Dreassi ${ }^{2}$, Sara Cornacchione ${ }^{3}$, Monica Tei ${ }^{3}$, \\ Francesca Coccina ${ }^{4}$ and Giuseppe Buonocore ${ }^{3}$ \\ ${ }^{1}$ Neonatal Intensive Care Unit, University Hospital, Siena, Italy; ${ }^{2}$ Department of Biotechnology and Chemistry and Pharmacy, University \\ of Siena, Siena, Italy; ${ }^{3}$ Department of Pediatrics, University of Siena, Siena, Italy; ${ }^{4}$ Department of Pediatrics, University of Chieti, \\ Chieti, Italy
}

\begin{abstract}
Background and objective: Acetaminophen is the active metabolite of phenacetin, a weak inhibitor of COX-1 and COX-2 in peripheral tissues, and it is one of the most widely prescribed drugs in pediatrics, used to weaken fever and to attenuate pain. Different formulations and dosages are available on the market. Division of the suppository form is a widely-used technique and it allows for optimization of the dosage based on the weight of the child. Yet, few data are available in the literature about the effectiveness of this practice. The aim of this study was to evaluate the uniformity of distribution of acetaminophen inside the suppository and to verify the homogeneity of distribution after the division.
\end{abstract}

Methods: Two hundred and twenty-four suppositories of different batches, dosages and producers were examined (Tachipirina ${ }^{\circledR}$, Efferalgan ${ }^{\circledR}$ ). One half of the suppositories $(n=112)$ were divided by the same operator in two halves along the vertical axis, and the other half $(n=112)$ were not divided but only weighed. In each of the groups (whole and fractional), the suppositories were evaluated by weight, acetaminophen content and the uniformity of the acetaminophen distribution.

Results: In no case did the partition of the suppositories led to significant differences ( $t$-test) between weight of the head and tail portion. No significant differences in weight, acetaminophen content or acetaminophen distribution of the active component were present in any of the two halves of the total suppositories analyzed after the partition.

Conclusions: Splitting acetaminophen suppositories is a common practice and it is a convenient medical procedure, with low chance of error, and it should not raise concerns of accurate dosage.

Introduction

Acetaminophen is the active metabolite of phenacetin, a weak inhibitor of COX-1 and COX-2 in peripheral tissues, and does not have significant anti-inflammatory effects. Paracetamol has been used in clinical practice for over 100 years. Acetanilid, the parent compound of paracetamol, was introduced in 1886. However, tox-

Keywords: Acetaminophen; Suppository; Paracetamol.

Abbreviations: $\mathrm{COX}$, cyclooxygenase; $\mathrm{AU}$, arbitrary unit; UV, ultraviolet.

Received: February 28, 2017; Revised: May 25, 2017; Accepted: May 26, 2017

${ }^{*}$ Correspondence to: Carlo Valerio Bellieni, Neonatal Intensive Care Unit, University Hospital "Le Scotte" Siena, Viale M Bracci 8, 53100, Siena, Italy. Tel: 00390577 586550, Fax: 00390577 586182, E-mail: cvbellieni@gmail.com

How to cite this article: Bellieni CV, Dreassi E, Cornacchione S, Tei M, Coccina F, Buonocore G. Accuracy in the Division of Acetaminophen Suppositories. Exploratory Research and Hypothesis in Medicine 2017;2(2):38-40. doi: 10.14218/ ERHM.2017.00003 icity-related problems with acetanilid lead to the introduction of paracetamol (acetaminophen, N- relationship between serum concentration and acetyl-p-amino-phenol) by von Mering in $1893 .{ }^{1}$

Nowadays, acetaminophen is one of the most widely prescribed drugs in pediatrics, in which it is used to weaken fevers and attenuate pain ${ }^{2}$; its frequency of use is followed by ibuprofen. Acetaminophen is well tolerated, lacks many of the side effects of aspirin, is available without prescription and is already widely used in the management of children with pain or fever. ${ }^{1}$

Current evidence suggests that there is no substantial difference in the safety and effectiveness of acetaminophen and ibuprofen in the care of a generally healthy child with fever. There is evidence, however, that combination of these two products is more effective than their use as single agents. Yet, there are concerns that combined treatment may be more complicated and contribute to the unsafe use of these drugs. Pediatricians should promote patient safety by advocating for simplified formulations, dosing instructions, and dosing devices. ${ }^{3}$ 


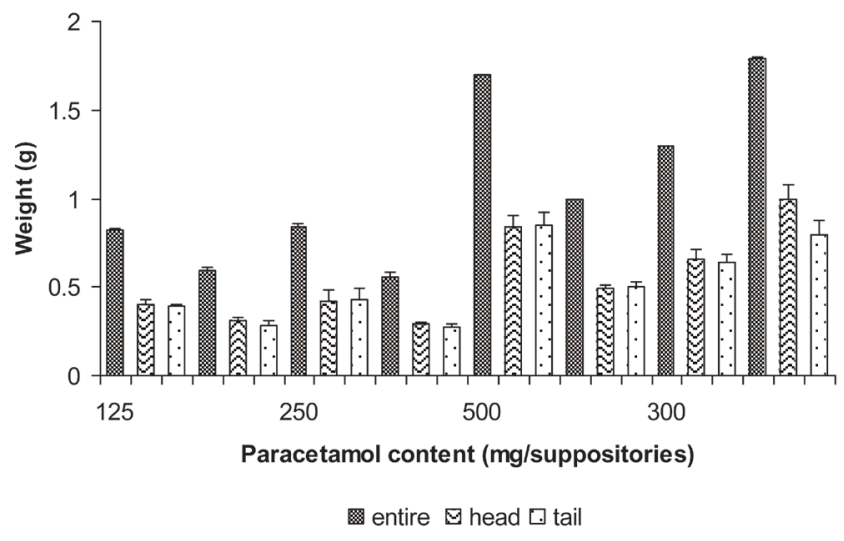

Fig. 1. Results for mean weigh \pm standard deviation of suppositories, including entire suppositories and those partitioned into head and tail portions.

Many products containing acetaminophen, with different formulations and concentrations, are available on the market. ${ }^{4}$ In most cases, acetaminophen is administered orally, while rectal administration is used for the treatment of fever in vomiting patients or in circumstances where oral administration is otherwise not possible. ${ }^{2}$

A wide number of rectal drugs, with various dosages $(125 \mathrm{mg}$, $150 \mathrm{mg}, 250 \mathrm{mg}, 300 \mathrm{mg}, 500 \mathrm{mg}, 1000 \mathrm{mg}$ ), are available on the market. The division of a rectal suppository is a widely-used technique and it allows for optimization of dosage that is administered according to the weight of the child, but few data are present in the literature regarding the efficacy of such a practice. ${ }^{5}$

\section{Study objective}

The objectives of this study were to evaluate the uniformity of distribution of acetaminophen inside suppositories of the investigated brands and to verify the homogeneity of distribution after the division. In fact, positioning the suppositories in a steady position for long time during the production or the storage period might provoke an attraction toward the base of their components, due to gravity, which might differ for several reasons, such as viscosity or hydrosolubility.

\section{Methods}

\section{Solvents and reagents}

All solvents and reagents were from BHD (Poole, England). Standard acetaminophen came from Sigma-Aldrich Srl (Milan, Italy). All commercial brand suppositories containing acetaminophen sold in Italy $(125,150,250,300$ and $500 \mathrm{mg})$ were purchased in local drugstores.

\section{Sample preparation}

Two hundred and twenty-four suppositories of different batches, dosages and producers were examined (Tachipirina ${ }^{\circledR}$ Angelini, Efferalgan ${ }^{\circledR}$ Bristol Myers). One half $(n=112)$ of the suppositories were divided by the same operator into two halves along the vertical axis; the two halves (head and tail) were then weighed. The

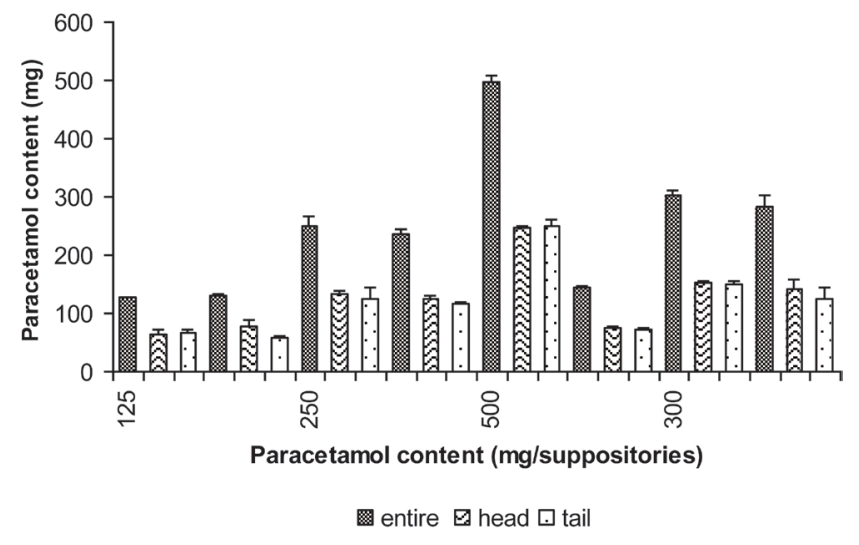

Fig. 2. Results for mean acetaminophen content \pm standard deviation of suppositories, including entire suppositories and those partitioned into head and tail portions.

other half $(n=112)$ of the suppositories were not divided, but only weighed.

Acetaminophen content was measured by UV-Vis spectroscopy. Each suppository, from both the whole or divided groups, were dissolved in a dichloromethane/isopropanol mixture (95:5 v/v) after weighing. All samples were diluted with the same solvent mixture to have an UV absorption at $248 \mathrm{~nm}$ in the range $0.4-0.7 \mathrm{AU}$.

The partition of the suppositories was realized with the same technique that would be used in practice to yield one half the value of the dose. The accuracy in partition was evaluated through comparison of the weight of the two portions of the suppositories (head and tail), its acetaminophen content and the uniformity of its distribution.

\section{UV analysis}

Suppositories (entire, head or tail) were dissolved and diluted by the aforementioned method, and the acetaminophen content was estimated through a calibration curve created on the basis of scalar solution in a dichloromethane/isopropanol mixture $(95: 5 \mathrm{v} / \mathrm{v})$ in the range $0.002-0.025 \mathrm{mg} / \mathrm{mL}$.

\section{Results}

The partition of the suppositories was simple to obtain, and the two portions (head and tail) retained their integrity so that they could be administered to a patient.

The results obtained for uniformity of weight of the various suppositories (entire, head and tail) are summarized in Figure 1. In no instance did the partition of the suppositories led to significant differences ( $t$-test) between weights of the head and tail portions.

In view of the composition of all analyzed suppositories (acetaminophen in synthetic triglycerides), the determination of the active component was carried out by UV spectroscopy, since the excipients did not show significant absorption at the wavelength used (248 $\mathrm{nm}$ maximum absorption of acetaminophen in dichloromethane/isopropanol solution). The results obtained for acetaminophen content in the various suppositories (entire suppository, head and tail) are summarized in Figure 2. All the suppositories analyzed (at least 10 samples from two production lots for each type) showed good uniformity of content (deviation from the theoretical value in the range $\pm 10 \%$ ). In no instance did the partition led to significant 


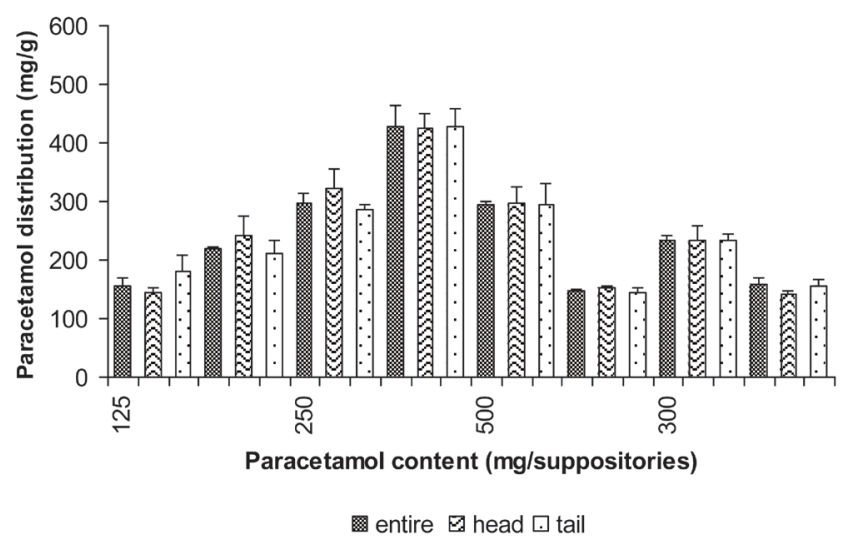

Fig. 3. Results for mean acetaminophen distribution \pm standard deviation of suppositories, including entire suppositories and those partitioned into head and tail portions.

differences ( $t$-test) between acetaminophen content of the head and tail portions.

The distribution of acetaminophen in the suppositories was determined with consideration to the weight of the sample (entire, head or tail portion), and the results are summarized in Figure 3. All the suppositories analyzed, of each brand investigated, showed a good homogeneity. In no case did the partition led to significant differences $(t$-test) between acetaminophen distribution of the head and tail portions.

\section{Discussion}

Partitioning acetaminophen suppositories is a common practice, but there are few data in the literature regarding the correctness and appropriateness of this kind of procedure.

Our study has shown that partitioning suppositories can be effective, guaranteeing the desired dose, since both halves retain their integrity, so that they can be safely administered to patients; in fact, no significant differences in weight, acetaminophen content and acetaminophen distribution were present in the two halves of the suppositories tested after splitting.

Our results are coherent with those of other studies which concluded that the analyzed commercial suppositories containing acetaminophen are of good quality in regard to homogeneity of content of the active ingredient. However, those authors concluded that the accuracy of partitions is unsatisfactory for achieving the target dose, and that only the whole suppositories were certain to contain the target dose. ${ }^{5}$

In our investigations, partitioning of the suppositories gave better results, with only 3 portions out of 328 ( 1 head and 2 tails) showing deviations from the target dose by more than $10 \%(+32.77 \%$, $-27.91 \%$ and $-19.26 \%$ of the target dose, respectively). This study was limited, however, in its ability to establish if this deviation is due to human error during the suppository portioning or if it is a product deviation.

\section{Future research directions}

Splitting suppositories is a viable medical procedure, since chance of error is very low and very much operator-related. Considering the homogeneous distribution of the drug inside the suppositories, each portion contains proportionally the right amount of active ingredient.

The problem of dividing a tablet or a suppository accurately is not limited to acetaminophen, but involves other drugs, in particular in pediatrics. A tight collaboration between pharmacologists and pediatricians is required, because when using drugs for infants or children that are otherwise formulated for adults it may become necessary to divide them, and it is indispensable to be aware of doing the right thing.

\section{Conclusions}

A manual split of a paracetamol suppository can be accurate and safe enough to be performed routinarely.

\section{Conflict of interest}

The authors have no conflict of interests related to this publication.

\section{Author contributions}

Performing the analysis of the suppositories (ED); coordinating the study $(\mathrm{CVB})$; collecting the literature and writing the paper (SC, MT, FC); revising the study (GB).

\section{References}

[1] Anderson BJ. What we don't know about paracetamol in children. Paediatr Anaesth 1998;8(6):451-460. doi:10.1046/j.14609592.1998.00295.x.

[2] Ohlsson A, Shah PS. Paracetamol (acetaminophen) for prevention or treatment of pain in newborns. Cochrane Database Syst Rev 2016;10:CD011219. doi:10.1002/14651858.CD011219.pub3.

[3] Section on Clinical Pharmacology, Therapeutics, Committee on Drugs, Sullivan JE, Farrar HC. Fever and antipyretic use in children. Pediatrics 2011;127(3):580-587. doi:10.1542/peds.2010-3852.

[4] Paracetamol. Available from: https://www.drugs.com/ingredient/ acetaminophen.html.

[5] Szostak R, Mazurek S. Quantification of active ingredients in suppositories by FT-Raman spectroscopy. Drug Test Anal 2013;5(2):126-129. doi:10.1002/dta.379. 